J. clin. Path. (1948), 1, 157.

\title{
THE USE OF INTRATHECAL HEPARIN IN CONJUNCTION WITH STREPTOMYCIN IN THE TREATMENT OF TUBERCULOUS MENINGITIS
}

\author{
PRELIMINARY REPORT
}

\author{
BY \\ CHARLES A. ST. HILL, CLIFFORD RILEY, AND J. HAMILTON GIFFORD \\ From the Royal Southern Hospital and the Fazakerley Sanatorium, Liverpool
}

(RECEIVED FOR PUBLICATION, FEBRUARY 10, 1948)

One of the commonest causes of failure in the treatment of tuberculous meningitis by streptomycin is the formation of intracranial and intraspinal adhesions with resultant hydrocephalus. These adhesions are produced partly as a result of the fibroblastic reaction that occurs around tuberculous foci, but their inception and growth may well be stimulated by the deposition of fibrin from the cerebrospinal fluid which is rich in fibrinogen in this condition. The photomicrograph (Plate II $a$ ) illustrates that fibrin is, in fact, laid down in this condition. This was taken from the spinal cord of a child who had died from tuberculous meningitis after several weeks of treatment by streptomycin, and the masses of fibrin in the upper part of the picture can be clearly seen. If fibrin deposition is of importance in the formation of tuberculous adhesions, therefore, it might reasonably be hoped that the prognosis would impiove if this could be prevented.

The obvious drug for this purpose is heparin. We decided for this reason to investigate the possibility of introducing this substance into the cerebrospinal fluid with streptomycin, and to attempt to assess its value in the treatment of tuberculous meningitis. Our preliminary findings on the interaction of streptomycin and heparin appeared to be of sufficient interest to warrant this preliminary communication. The investigations so far completed, and the conclusions derived therefrom, are given below.

\section{Effects of Admixture of Heparin and Streptomycin Solutions}

When a solution of heparin ("liquemin," or " heparin Boots," or " heparin Evans") was added to one of commercial streptomycin (hydrochloride or calcium complex), a precipitate was slowly formed. The nature of this precipitate has not yet been fully investigated, but certain properties have been noted. Examination of the supernatant fluid after centrifugalization revealed that there was no diminution of the streptomycin content during this reaction. Streptomycin assays were carried out both by serial dilutions in fluid media and cup plate methods.

The results of one of the latter methods of assay are shown in Plate II $b$. In this experiment, one drop of streptomycin solution of strength 25 units per $\mathrm{ml}$. was added in the cup $C$ to one drop of heparin solution of strength 1,000 units per ml., thus ensuring that a very large excess of the latter was present. The photograph shows that there was no diminution of the area of clearance of the test organism by this mixture as compared with the control cup B, into which was placed one drop of the same streptomycin solution and one drop of saline. Into cup $\mathbf{A}$ was placed one drop of a 1,000 unit per ml. heparin solution in order to demonstrate that it has no bacteriostatic powers.

Solutions of known strengths of heparin and streptomycin were again mixed and, after centrifugalization, the heparin contents of the supernatant fluid and of the deposit were estimated.

In the earlier stages of the work this heparin content was assayed on the basis of its delaying powers upon the deposition of fibrin from human plasma in the presence of excess thrombokinase and adequate calcium. This method is based on the assumption that heparin exerts its influence by the inactivation of thrombin and prothrombin (Best and Taylor, 1945). As McIntosh (1941) has pointed out, however, this method tends to give inconsistent results so that it was later replaced by a slight modification of the toluidine blue method of Trethewie and Melvin (1945). We have compared the colours of the unknown heparin toluidine blue mixture with that of the standard in a colorimeter instead of in comparator tubes as described by them. This method is quicker and gives consistent results but, of course, measures heparin in colour units. 
These assays showed that:

(a).-If the amount of heparin in solution (in mg.) was less than approximately two-thirds of the amount of streptomycin (also in mg.) to which it was added, then no heparin was present in the supernatant fluid after removal of the precipitate. This was true whether the heparin was assayed by the toluidine blue method or by its anticoagulatory effect. This applies to the commercial preparations of heparin used by us, which contained between 90 and 110 units of heparin per mg.

(b).--If more heparin was then added to the mixture a further precipitation occurred, but active heparin began to appear in the supernatant fluid, so that by adding sufficient excess of heparin, concentrations of the order of 1,000 units per ml. or higher could be obtained free in the clear supernatant fluid in the presence of streptomycin. Even when these concentrations of heparin had been obtained, the addition of further heparin to the mixture still produced a precipitate. The addition of more streptomycin, on the other hand, did nct cause a precipitate. Thus it was possible to prepare a mixture of heparin and streptomycin in clear solution and high concentration which could be introduced into the theca even though previously injected streptomycin was still present, without the danger that an intrathecal precipitation might $\stackrel{\stackrel{0}{\vec{\sigma}}}{\frac{0}{0}}$ occur.

(c).-The precipitate, which consisted of a mix- $\stackrel{\vec{F}}{\stackrel{P}{+}}$ ture of a white powdery material and a black tarry? substance, contained active heparin as revealed both by the toluidine blue method and its anti- $\frac{\bar{N}}{7}$ coagulatory effect. As no streptomycin was lost $\stackrel{\mathbb{Q}}{\mathscr{Q}}$ from solution, there was presumably no streptomycin in the precipitate. Thus the precipitate must $\vec{\partial}$ have been produced by a reaction between heparin and some impurity in the streptomycin.

(d).-All the heparin lost from the supernatant $\stackrel{ }{\circ}$ fluid was found to be present in the precipitate.

(e).-The precipitate showed slight solubility when warmed.

Effect of Introducing Heparin into the Theca of $v$ a Patient receiving Intrathecal Streptomycin

This was only tried on one patient, owing to the $\vec{r}$ adverse reaction obtained. One $\mathrm{ml}$. of a 1,000 unit per ml. solution of heparin was introduced into the theca daily, immediately after the introduction $\odot$ of streptomycin, and at the end of the twenty-four- $\infty$ hour period the streptomycin and heparin contents of the cerebrospinal fluid were assayed. The changes in the cerebrospinal fluid and the reaction of the patient are given in the composite graph
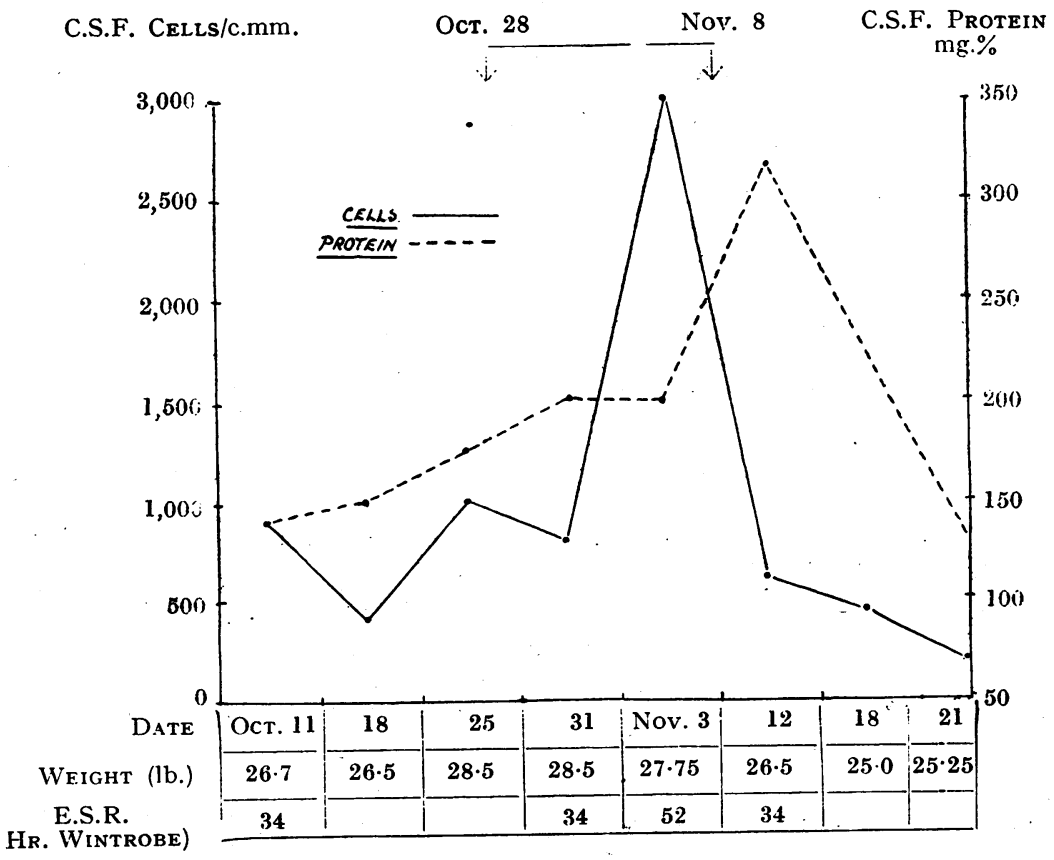

Fig. 1.-Changes in the cerebrospinal fluid, weight, and erythrocyte sedimentation rate during a course of daily intrathecal injections of heparin and streptomycin given separately. From Oct. 28 to No\% 8 the patient had been receiving intrathecal and intramuscular streptomycin. 
(Fig. 1). As can be seen from this, the white cells and protein contents of the cerebrospinal fluid rose sharply to 3,000 per c.mm. and $320 \mathrm{mg}$. per $100 \mathrm{ml}$. respectively. Coinciding with this, the patient began to show neck rigidity and incontinence with increased irritability and anorexia, whilst his weight, which had previously been increasing, started to decrease. On several occasions he showed evidence of experiencing pain immediately after the heparin injections, but it was impossible to confirm this as he was not co-operative.

This single case suggested that this method of giving heparin was not practicable. A combined solution of heparin and streptomycin was therefore prepared by adding 80,000 units of heparin in $10 \mathrm{ml}$. of saline to $1,000,000$ units $(1 \mathrm{~g}$.) of streptomycin in an equal volume of saline. The mixture was allowed to stand in the refrigerator overnight to allow the maximum deposition of precipitate. The clear supernatant fluid was then decanted. The streptomycin content of this clear fluid was 50,000 units per ml., whilst the heparin content was found to be approximately 1,000 units per $\mathrm{ml}$. This was injected in $2.0 \mathrm{ml}$. doses intrathecally into the same patient who had previously reacted adversely to the separate injections of streptomycin and heparin. The graph (Fig. 2) shows that the reaction produced was slight. We were thus encouraged to repeat the process in two other patients who had just been admitted and who had received no streptomycin previously. The changes in the cerebrospinal fluid of one of these are shown in Fig. 3 and, as can be seen, there was very little adverse reaction. The dose of free heparin was later increased to 5,000 units per dose in order to produce a residual concentration of between 1 and 2 units of heparin per ml. of cerebrospinal fluid at the end of the twenty-four-hour period.

Effect upon Heparin of the Streptomycin of the Cerebrospinal Fluid, following Purely Intramuscular Injections of this Latter Substance

It would appear probable that the precipitate formed by the admixture of solutions of heparin

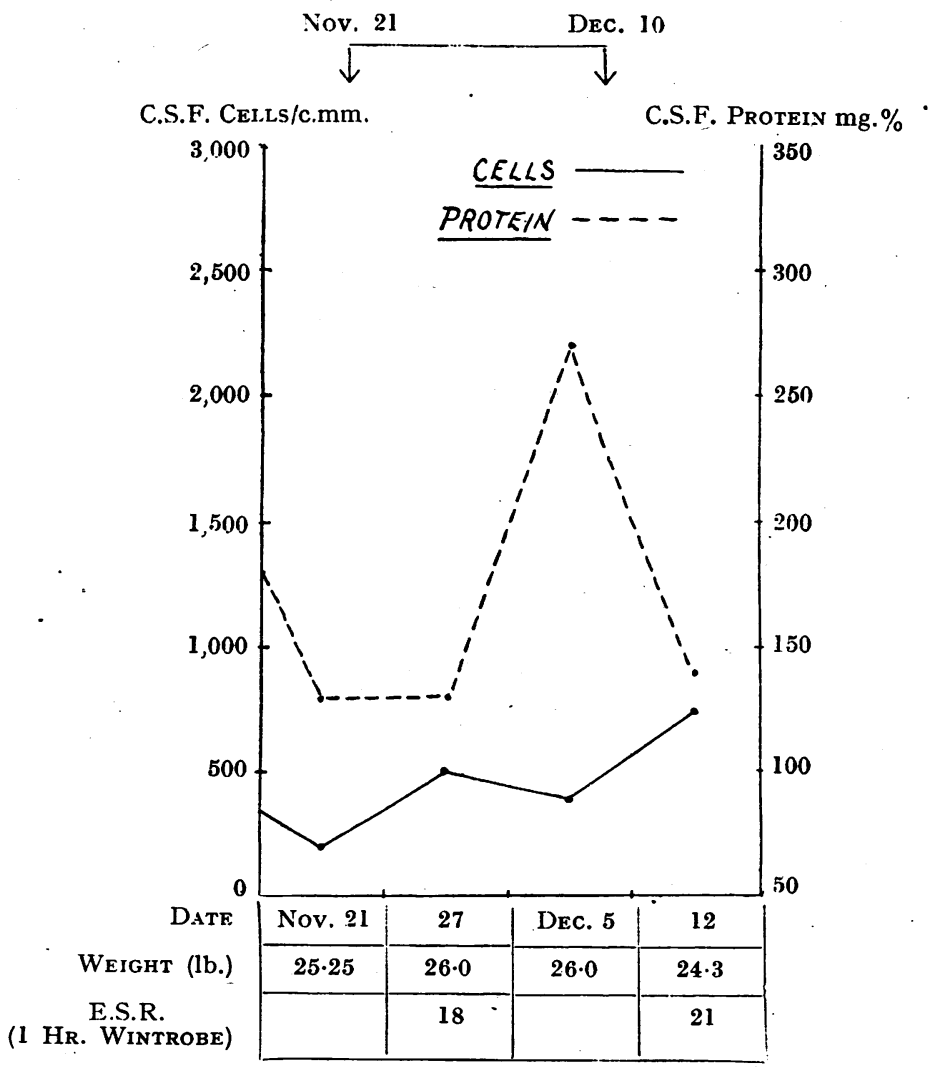

FIG. 2.-Cerebrospinal fluid changes during a course of daily injections of heparin-streptomycin mixture from Nov. 21 to Dec. 10. Case 1. 


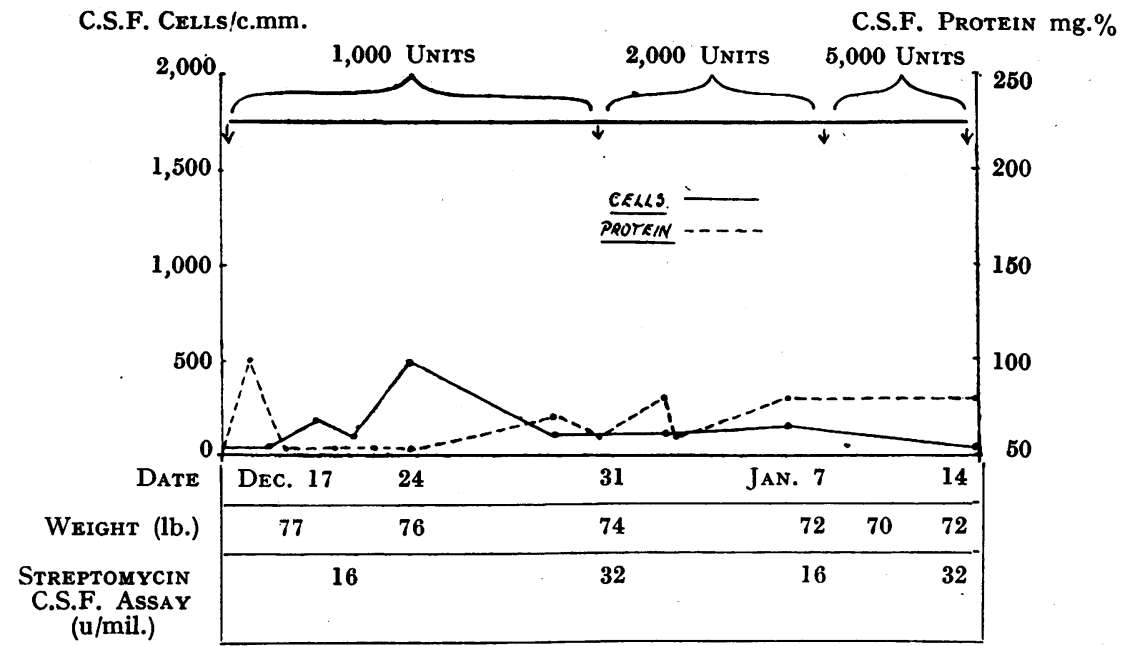

Fig. 3.-Cerebrospinal fluid changes during the daily administration of heparinstreptomycin mixture containing a higher concentration of heparin than in Case 1.

and streptomycin is caused by impurities in the streptomycin. It was decided, therefore, to determine whether streptomycin that has been given intramuscularly and has reached the cerebrospinal fluid via the blood stream has a similar effect upon heparin solutions, that is, the production of a precipitate. Specimens of cerebrospinal fluid were obtained from patients receiving streptomycin by the intramuscular route only. These were withdrawn about one hour after the intramuscular streptomycin had been given, so that the concentration of the drug should be at a maximum. Equal quantities of this and of known strengths of heparin solutions were mixed, allowed to stand for twelve hours, centrifugalized, and the surviving heparin assayed. It was found that in all cases a small proportion of the heparin was lost, but that this was not related to the amount of streptomycin present in the cerebrospinal fluid samples It may well be that the losses that occurred were due to fixation of heparin by fibrin precursors of the cerebrospinal fluid.

Effect of Injecting Heparin into the Theca of Patients receiving Intramuscular Streptomycin Only

The evidence derived from the preceding experi ment suggested that no adverse reaction would 
follow the intrathecal administration of heparin to a patient who was receiving streptomycin by the intramuscular route only. Heparin was introduced, therefore, in 2,000-unit doses daily into the theca of a patient receiving this form of therapy, and changes in his cerebrospinal fluid and clinical condition were noted. The graph (Fig. .4) shows that no significant adverse reaction occurred.

\section{Conclusions}

1. The admixture of streptomycin and heparin causes the appearance of a precipitate which removes a large proportion of the heparin from solution, but has no effect on the streptomycin.

2. The occurrence of this reaction in the spinal theca produces an adverse effect on the patient.

3. A mixture of streptomycin and heparin in clear solution can be prepared which can be introduced with safety into the theca and gives comparable streptomycin assays with those found if streptomycin is given alone.

4. When the patient is being given intramuscular streptomycin only, heparin can be introduced into the theca without causing a reaction.

By this means it is possible to inject heparin regularly into the thecae of patients suffering from tuberculous meningitis under streptomycin treatment in an attempt to delay or inhibit the formation of adhesions. The effect of this on prognosis has yet to be investigated.

\section{Addendum}

Since sending this article to press we have been receiving samples of very pure heparin from the Evans Biological Institute, Runcorn. Very little precipitation results from the admixture of solutions of this heparin and streptomycin, and the supernatant fluid contains a very high concentration of heparin. This has resulted in a saving of heparin, but accentuates the need for assaying the heparin content of all heparin/streptomycin mixtures before use.

Our thanks are due to Dr. A. C. T. Vaughan for the streptomycin assays of cerebrospinal fluids; to Dr. R. R. Hughes for permission to investigate his patients; to Dr. J. M. Swithinbank for his willing co-operation in treating the cases; and to Drs. O. F. Thomas and A. B. Christie for providing beds for the patients under investigation. The streptomycin used in this work was provided by the Streptomycin Committee of the Ministry of Health.

\section{REFERENCES}

Best, C. H., and Taylor, N. B. (1945). "The Physiological Basis of Medical Practice." 4th ed., p. 87. London.

McIntosh, F. C. (1941). Biochem. J., 35, 776.

Trethewie, F. C., and Melvin, P. A. (1945). Austral. J. exp. Biol., 23,241 . 